\title{
$S U(N)$ 规范场静态球对称双子解
}

\author{
马中 骐 \\ (中国科学院高能物理研究所)
}

几年前, 'tHooft 和 Polyakov ${ }^{[1]}$ 找到了 $S U(2)$ 规范场能量有限的磁单极正则解. 他们讨 论 $S U(2)(S O(3))$ 规范场和属伴随表示的 Higgs 场的相互作用系统, 假设（ansatz）了狭义 静态和同步球对称的规范势和 Higgs 场的标准形式,代人杨-Mills ${ }^{[2]}$ 方程,得到所含函数满足 的常微分方程. 联立非线性常微分方程组仍难求解，而在无穷远处，Higgs 场趋于 Higgs 真 空,方程组化为代数方程, 可解得点磁单极解即吴-杨解 ${ }^{\left[{ }^{[3]}\right.}$, 然后他们以此为边界条件, 选择试 验函数, 代人能量表式进行变分, 得到能量有限的磁单极正则解和磁单极的总能量 (质量). Prasad-Sommerfield ${ }^{[4]}$ 在保持 Higgs 场真空破缺而让作用势 $V(\phi)$ 趋于零的极限 $(P-S$ 极限) 下, 得到能量有限的磁单极解析解, 后来证明 ${ }^{[5]}$ 这是在 $P-S$ 极限下能量最低的磁单极解, 这能 量往往用来估计磁单极的质量 ${ }^{[6]}$. 通过 Julia-徐 ${ }^{[7]}$ 变换得到双子（Dyon）解, 在这解中 $W^{0}$ 与 Higgs 场 $\phi$ 成比例, 但这并非双子解必须满足的条件. 由于 $S U(2)$ 静态球对称规范场满足的 常微分方程组已经得到, 作为无穷远边界条件的点双子解不难解得 ${ }^{[8]}$, 通过 'tHooft 的变分方 法,同样可以得到能量有限的正则双子解.

有不少人试图把 $S U(2)$ 磁单极解和双子解推广到 $S U(N)$ 规范场中去 ${ }^{[8-11]}$, 按照 'tHooftPolyakov $^{[1]}$ 的方法, 关键在于找出正则解在无穷远的边界条件, 即所谓点磁单极解和点双子解. 方法主要有两类. 一类以 Wilkinson-Goldhaber ${ }^{[9]}$ 为代表, 假定点磁单极解都可以通过奇异规 范变换化到带奇异弦的阿贝尔规范去, 在作了某些假设 (ansatz) 以后, 证明了在阿贝尔规范 下点磁单极解的一般形式, 并找到了变回无弦规范的奇异规范变换. 他们没能导出静态球对 称规范势满足的常微分方程组, 因此无法讨论 $S U(N)$ 点双子解的一般形式 ${ }^{[8]}$. 另一类以 Corrigan-Olive-Fairlie-Nuyts ${ }^{[10]}$ 为代表，他们希望直接在无弦规范中找出静态球对称规范势的 一一般形式及其运动方程, 然后解出作为 $r \rightarrow \infty$ 边界条件的点磁单极解. 但因未找到有效的 二[具, 他们只处理了 $S U(3)$ 规范场, 向 $S U(N)$ 推广很困难. 我们运用谷超豪提出的标准微 分环路位相因子的方法 ${ }^{[12]}$, 放弃上述两类方法都采用的那些假设,找到 $S U(N)$ 静态球对称规 范势的一般形式及其满足的常微分方程组, 作为能量有限的磁单极解在无穷远的边界条件,严 格地解出 $S U(N)$ 全部不等价的静态球对称点磁单极解 ${ }^{[13]}$. 由于有了运动方程组, 原则上可 以解出全部点双子解. 本文运用前文 ${ }^{[12-13]}$ 的方法, 作为能量有限的双子解在无穷远边界条件, 解出全部 $S U(N)$ 静态球对称点双子解.

采用前文 ${ }^{[13]}$ 的符号

$$
\boldsymbol{W}=-\frac{1}{e r} \sum_{j a} \Gamma_{a}^{j} \hat{\boldsymbol{M}}_{a}^{i j}+\frac{1}{e r} \sum_{j k^{a}}\left[\hat{\boldsymbol{L}}_{a}^{j k} \mathrm{Re}+\hat{M}_{a}^{j k} \operatorname{Im}\right] w_{a}^{j k},
$$

本文 1981 年 12 月 7 日收到.

第 10 期

科学通报 


$$
\begin{aligned}
& W^{i}=\frac{1}{e r} \cdot \sum_{j h a}\left[N_{i:}^{i k} \operatorname{Re}+\widetilde{N}_{a}^{i k} \cdot \operatorname{Im}\right] P_{i,}^{i k}, \\
& \phi=\frac{1}{e r} \sum_{j k a}\left[N_{a}^{j k} \operatorname{Re}+\widetilde{N}_{a}^{j k} \operatorname{Im}\right] H_{a}^{j k}, \\
& \Gamma_{a}^{j}=\sqrt{(j+a)(j-a+1)}, \quad w_{a}^{j k}=u_{a}^{i k}+i v_{a}^{j k}, \\
& P_{a}^{i k}=p_{a}^{i k}+i \tilde{p}_{a}^{j k}=P_{a}^{k j *}, \quad H_{a}^{j k}=h_{a}^{j k}+i \tilde{h}_{a}^{j k}=H_{a}^{k} ; *, \quad j
\end{aligned}
$$

在 $r \rightarrow \infty$ 时, Higgs 场趋于 Higgs 真空

$$
V^{\prime}(\phi)=0, V(\phi)=0, \quad D_{\mu} \phi=0 .
$$

由于无穷远处无广义电荷, 场强应沿径向, 且不慢于 $\frac{1}{r^{2}}$ 趋于零:

$$
\boldsymbol{\delta}=\hat{\boldsymbol{r}} O\left(\frac{1}{r^{2}}\right), \quad \mathscr{B}=\hat{\boldsymbol{r}} O\left(\frac{1}{r^{2}}\right),
$$

取到 $\frac{1}{r}$ 最低次项

$$
\phi_{0} S_{a}^{j k} \equiv \frac{H_{a}^{i k}}{e r}=\phi_{0} S_{i}^{j k}(t), \quad w_{a}^{j k}=w_{a}^{j k}(t), \quad P_{a}^{i k}=P_{a}^{(0) i k}(t) r+p_{a}^{(1) i k}(t),
$$

取到同级近似,必须保留 $P_{a}^{(1) i k}$ 的项*,场强 8 主要来自它的贡献, $P_{a}^{(0) i_{k}}$ 项对场强径向分量无贡 献. 我们的任务就是要解出这些量的可能取值. 前文 ${ }^{[33]}$ 曾假定 $P_{a}^{i k}=0$, 得出点源解一定是 狭义静态的结论，但 $P_{a}^{(0) i k} \neq 0$ 时这结论不再成立. 静态球对称规范势随时间一般依赖关系 为 ${ }^{[13]}$

因此

$$
\begin{gathered}
W_{\mu}(\boldsymbol{r}, t)=T(t) W_{\mu}(\boldsymbol{r}, 0) T^{-1}(t), \quad \phi(\boldsymbol{r}, t)=T(t) \phi(\boldsymbol{r}, 0) T^{-1}(t), \\
T(t)_{a, b_{k}}=\delta_{j k} \delta_{a b} e^{i \alpha_{j} t}, \quad \sum_{i} \boldsymbol{\alpha}_{j}(2 j+1)=0,
\end{gathered}
$$

$$
\begin{gathered}
w_{a}^{i k}(r, t)=e^{i\left(\alpha_{j}-\alpha_{k}\right)^{t} w_{a}^{i k}(r, 0),} \\
P_{a}^{i k}(r, t)=e^{-i\left(\alpha_{j}-\alpha_{k}\right) t} P_{a}^{i k}(r, 0), \quad H_{a}^{i k}(r, t)=e^{-i\left(\alpha_{j}-\alpha_{k}\right) t} H_{a}^{i k}(r, 0),
\end{gathered}
$$

限于无穷远球面上, 把点源解看成规范势, 作规范变换 $T^{-1}(t)$, 得

$$
\begin{aligned}
& w_{a}^{i k}(t) \rightarrow w_{a}^{j k}(0), \quad S_{a}^{j k}(t) \rightarrow S_{a}^{i k}(0), \\
& P_{a}^{(0) i_{k}(t)} \rightarrow P_{a}^{(0) i k}(0)-\alpha_{j} \delta_{i k}, \quad P_{a}^{(1) j k}(t) \rightarrow P_{a}^{(1) ; k}(0) .
\end{aligned}
$$

与前文 ${ }^{[13]}$ 讨论的小群变换一样,经过此变换前后的解都可作为点源解, 即作为标准微分环路位 相因子在无穷远的渐近形式, 但是作为位相因子,变换前后的解是不等价的. 这类变换 $T((t)$ 是另一种类型的小群变换，经此变换前后的点源解互相准等价,任何点源解都准等价于与时间 无关的狭义静态点源解. 对后者, 代人运动方程得 ${ }^{[13]}$

$$
\begin{aligned}
& \boldsymbol{\delta}=-\hat{\boldsymbol{r}} \sum_{i k a}\left[N_{a}^{i k} \operatorname{Re}+\widetilde{N}_{a}^{i k} \operatorname{Im}\right]\left(\frac{P_{a}^{i k}}{e r}\right)^{\prime} \\
& +\frac{1}{e r^{2}} \sum_{i k a}\left[-\hat{\boldsymbol{L}}_{a}^{i k} \operatorname{Im}+\hat{\boldsymbol{M}}_{a}^{i k} \operatorname{Re}\right] \sum_{i}\left(P_{a}^{i t *} w_{a}^{t k}-w_{a}^{i t} P_{a-1}^{t k *}\right), \\
& \mathscr{B}=\frac{\hat{\boldsymbol{r}}}{e r^{2}} \sum_{j a} a N_{a}^{j j}+\frac{\hat{\boldsymbol{r}}}{2 e r^{2}} \sum_{j k a}\left[N_{a}^{j k} \operatorname{Re}+\widetilde{N}_{a}^{j k} \operatorname{Im}\right] \sum_{i}\left(w_{a+1}^{t j} w_{a+1}^{t k *}-w_{a}^{j t *} w_{a}^{k t}\right),
\end{aligned}
$$

* 严格讲, 由于 $P^{(0)}$ 项的存在, 在有的地方还需引人 $P^{(2)} \frac{1}{r}$ 的项, 但此项对总电荷没有贡献, 而且文中的各结论都依 然成立, 这里不仔细讨论了。 


$$
\begin{aligned}
& D^{0} \phi=\frac{1}{e r^{2}} \sum_{i k a}\left[-N_{a}^{i k} \operatorname{Im}+\widetilde{N}_{a}^{i k} \operatorname{Re}\right] \sum_{t}\left(P_{a}^{i t} H_{a}^{t k}-H_{a}^{i t} P_{a}^{t k}\right)=0, \\
& \boldsymbol{D}_{\phi}=-\hat{\boldsymbol{r}} \sum_{j k^{a}}\left[N_{a}^{i k} \operatorname{Re}+\tilde{N}_{a}^{i k} \operatorname{Im}\right]\left(\frac{H_{a}^{j k}}{e r}\right)^{\prime} \\
& +\frac{1}{e r^{2}} \sum_{j k a}\left[-\hat{\boldsymbol{L}}_{a}^{i k} \operatorname{Im}+\hat{\boldsymbol{M}}_{a}^{i k} \operatorname{Re}\right] \sum_{t}\left(H_{a}^{j t *} w_{a}^{i k}-w_{a}^{j \ell} H_{a-1}^{i k *}\right)=0, \\
& J^{0}=\frac{1}{e r^{3}} \sum_{i k a}\left(N_{a}^{i k} \operatorname{Re}+\widetilde{N}_{a}^{i k} \operatorname{Im}\right) \sum_{i i}\left[w_{a+1}^{t i}\left(w_{a+1}^{i *} P_{a}^{i k}-P_{a+1}^{t i} w_{a+1}^{i k *}\right)\right. \\
& \left.+w_{a}^{i t *}\left(w_{a}^{i t} P_{a}^{k i *}-P_{a-1}^{i \tau *} w_{a}^{k i}\right)\right]=0 \text {, } \\
& \boldsymbol{J}=\frac{\hat{\boldsymbol{r}}}{e r^{2}} \sum_{i k a}\left(-N_{a}^{i k} \operatorname{Im}+\tilde{N}_{a}^{i k} \operatorname{Re}\right) \sum_{t}\left[P_{a}^{i t}\left(P_{a}^{t k}\right)^{\prime}-\left(P_{a}^{i t}\right)^{\prime} P_{a}^{t k}\right] \\
& +\frac{1}{2 e r^{3}} \sum_{i k a}\left[\hat{\boldsymbol{L}}_{a}^{i k} \operatorname{Re}+\hat{\boldsymbol{M}}_{a}^{i k} \operatorname{Im}\right]\left\{-2 \boldsymbol{w}_{a}^{j k}+\sum_{i i}\left[2 w_{a}^{i i} w_{a}^{t i *} w_{a}^{i k}-w_{a}^{i j} w_{a-1}^{i t} w_{a-1}^{k t *}\right.\right. \\
& -w_{a+1}^{i *} w_{i+1}^{i i} w_{a}^{i k}+2\left(P_{a}^{i i *} w_{a}^{i t}-w_{a}^{i i} P_{a-1}^{i t *}\right) P_{a-1}^{t k *} \\
& \left.\left.+2 P_{a}^{i i *}\left(w_{a}^{i t} p_{a-1}^{t k *}-P_{a}^{i t *} w_{a}^{i k}\right)\right]\right\}=0 .
\end{aligned}
$$

由 $D^{0} \phi=0$ 和 $\boldsymbol{J} \cdot \hat{r}=0$ 得

$$
\begin{aligned}
& \sum_{t}\left[P_{a}^{(i) j t} S_{a}^{r k}-S_{a}^{j t} P_{a}^{(i) t_{k}}\right]=0, \quad i=0.1, \\
& \sum_{t}\left[P_{a}^{(1)_{i} t} P_{a}^{(0) t_{k}}-P_{a}^{(0){ }_{a} t} P_{a}^{(1) t k}\right]=0,
\end{aligned}
$$

可见 $\hat{\phi}\left(x_{0}\right), W^{(0)}\left(x_{0}\right)$ 和 $W^{(1)}\left(x_{0}\right)$ (分别与 $P^{(0)} 、 P^{(1)}$ 相对应) 可以互相对易, 因此它们可通过 小群变换同时对角化 ${ }^{[13]}$ (准等价):

$$
S_{a}^{j k}=\delta_{i k} S_{a}^{i j}=\text { 实数; } P_{a}^{i k}=\delta_{i k} P_{a}^{i j}=\text { 实数. }
$$

再由 $\boldsymbol{D}_{\phi}=0$ 和 $\boldsymbol{\varepsilon}$ 的切向为零, 得

$$
\left(S_{a}^{i j}-S_{a-1}^{k k}\right) w_{a}^{i k}=0, \quad\left(p_{a}^{(i) i j}-p_{a-1}^{(i) k k}\right) w_{a}^{i k}=0, \quad i=0.1,
$$

即

$$
w_{a}^{i k}=0 \text {, 当 } S_{a}^{i j} \neq S_{a-1}^{k k} \text { 或 } p_{a}^{(i) i j} \neq p_{a-1}^{(i) k k}, \quad i=0.1 \text {. }
$$

因此, $J^{0}=0$ 自动满足, 由 $\boldsymbol{J}=0$ 得

$$
-2 w_{a}^{i k}+\sum_{i i}\left[2 w_{a}^{j i} w_{a}^{i *} w_{a}^{* k}-w_{a}^{i i} w_{a-1}^{i t} w_{a-1}^{k t *}-w_{a+1}^{t ; *} w_{a+1}^{t i} w_{a}^{i k}\right]=0,
$$

此式与前文 ${ }^{[13]}$ 点磁单极解满足的公式完全一样, 按照那里给出的方法, 通过小群变换可以把 $w w_{a}^{i k}$ 化成

$$
\left.\begin{array}{c}
w_{a}^{i k}=i v_{a}^{i k}, \quad v_{a}^{i k} \geqslant 0, \\
v_{a}^{i k} v_{a}^{t k}=\delta_{j i}\left(v_{a}^{i k}\right)^{2}, \quad v_{a}^{j k} v_{a}^{j t}=\delta_{k i t}\left(v_{a}^{i k}\right)^{2} .
\end{array}\right\}
$$

若有 $m$ 个相连的 $v_{a}^{j k} \neq 0$ :

则

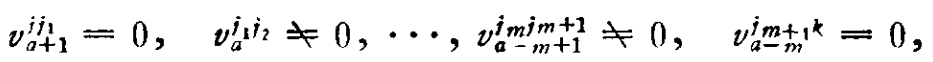

$$
\begin{aligned}
& v_{a}^{i_{n}{ }^{j} n_{n+1}+1}=\sqrt{n(m-n+1)} \text {. }
\end{aligned}
$$

由于 (10)式, 这种小群变换并不改变 $\hat{\phi}$ 和 $W^{\prime}$. 这样, 根据前文 ${ }^{[13]}$ 给出的点磁单极解, 按照 (10) 式补上相应的 $p^{(0) i j}$ 和 $p^{(1) i j}$, 就得到 $S U(N)$ 全部不准等价的静态球对称点双子解, 再通过小群 变换可以得到不等价的点双子解. 注意 $W^{0}$ 与 $\hat{\phi}$ 不同之处在于 $p_{a}^{i j}$ 不限于只取两种不同数值, 除 (10)式和无迹条件外, $p_{a}^{i j}$ 再无其它限制. 对无交叠情况,正如前文 ${ }^{[13]}$ 所讨论的, $w_{a}^{i k}$ 只能取 
纯虚数, $\hat{\phi}\left(x_{0}\right)$ 和 $W^{0}\left(x_{0}\right)$ 也自然是对角化的, 不存在上述的小群变换, 上法所得的就是全部不 等价的静态球对称点双子解.

以上述点双子解为边界条件的能量有限的双子解, 总磁荷仍如前文 ${ }^{[13]}$ 所给出的:

$$
\begin{aligned}
& g=\frac{4 \pi}{e} \sum_{j a}\left(2 a s_{a}^{i j}\right)=\sum_{a>0} \frac{2 a g_{0}}{A-B} \sum_{i}\left(s_{a}^{j i}-s_{-a}^{i j}\right), \\
& g_{0}=\frac{4 \pi}{e}(A-B), \quad A=\sqrt{\frac{N-n}{2 N n}}, \quad B=-\sqrt{\frac{n}{2 N(N-n)}},
\end{aligned}
$$

$S_{a}^{j i}$ 中有 $n$ 个取 $A,(N-n)$ 个取 $B$. 在无穷远处广义电场为

$$
\boldsymbol{\mathscr { e }}=\frac{\hat{\boldsymbol{r}}}{e r^{2}} \sum_{j a} N_{a}^{i j} p_{a}^{(1) i j},
$$

电场为

$$
\boldsymbol{E}=2 \operatorname{Tr}[\mathscr{E} \hat{\phi}]=\frac{2 \hat{\boldsymbol{r}}}{e r^{2}} \sum_{j a} p_{a}^{(1) i j} S_{a}^{j j}
$$

总电荷为

$$
q=\oiint d \boldsymbol{S} \cdot \boldsymbol{E}=\frac{8 \pi}{e} \sum_{j a} p_{a}^{(1) j i} s_{a}^{i j},
$$

加上半径典的量子化条件 ${ }^{[8]}$, 电荷才取量子化值. 在 $r \rightarrow \infty$ 处的能量密度为

$$
\theta_{00}=\operatorname{Tr}\left(\mathscr{B}^{2}+\mathscr{B}^{2}\right)=\frac{1}{4 e^{2} r^{4}} \sum_{j a}\left\{\left(2 p_{a}^{(1) i j}\right)^{2}+\left[2 a+\sum_{t}\left(v_{a+1}^{i j}\right)^{2}-\sum_{r}\left(v_{a}^{j t}\right)^{2}\right]^{2}\right\},
$$

$g 、 q$ 和 $\theta_{00}$ 都对小群变换保持不变, 即准等价的双子解有相同的磁荷、电荷和在无穷远处的能 量密度.

致谢：作者感谢胡宁教授和朱洪元教授的关心和鼓励.

参考文献

[ 1 ] 'Hooft. G., Nucl, Phys, B79 (1974), 276; Polyakov, A. M., JETP Lett., 20 (1974). 194.

[2] Yang, C. N. \& Mills, R. L. Phys. Rev., 96 (1954), 191.

[3] Wu, T. T. \& Yang, C. N., Properties of Matterunder Unusual Conditions (Eds. Mark. H. \& Fernbach, S.), New York, Interscience, 1969. 344,

[4] Prasad, M. K. \& Sommerfield, C. M., Phys. Rev. Lett., 35 (1975), 760.

[5 ] Coleman. S., Parke, S., Neveu. A. \& Sommerfield, C. M., Phys. Rev., D15 (1977), 544.

[6] Scott, D. M., Nucl. Phys., B171 (1980), 109.

[ 7 ] Julia, B. \& Zee, A., Plyys. Rev., D11 (1975), 2227.

[8] Dokos. C. P. \& Tomaras, T. N., Phys. Rev., D21 (1980), 2940.

[9] Wilkinson, D. \& Goldhaber, A. S., Phys. Rev., D16 (1977), 1221.

[10] Corrigan, E., Olive, D. I., Fairlie, D. B. \& Nuyts, J., Nucl. Phys., B106 (1976). 475.

[11] Marciano, W. J. \& Pagels, H., Phys. Rev, D12 (1975), 1093; Chakrabarti, A. Ann. Inst. H. Poinoare, 23 (1975), 235; Nucl. Phys., B101 (1975), 159; Wu, A. C. T. \& Wu, T. T., J. Math. Phys., 15 (1974), 53; Wilkinson, D \& Bais, F. A.. Phys. Rev., D19 (1979), 2410

[12] 谷超家, 复旦学报 (自然科学版)，1976、2:51；1977，2:30.

[13] 马中骐, BIHEP-TH-10，1981; 中国科学，A 辑，1982，5: 421. 